\title{
A HIGH PRECISION DEM EXTRACTION METHOD BASED ON INSAR DATA
}

\author{
Wang Xinshuang ${ }^{1}$, Liu Lingling ${ }^{1}$, Shi Xiaoliang ${ }^{2, *}$, Huang Xitao ${ }^{1}$, Geng $\mathrm{Wei}^{1}$ \\ ${ }^{1}$ Shannxi Geomatics Center of National Administration of Surveying, Mapping and Geoinformation, Xi'an, China - (shuang1007, \\ lingling600650, huangxitao_81,geng_wei0728)@163.com \\ ${ }^{2}$ College of Geomatics, Xi'an University of Science and Technology, Xi'an, China - shixiaoliang0305@gmail.com
}

\section{Commission III, WG III/2}

KEY WORDS: InSAR, DEM, Interferometric, Adaptive Filtering, Phase Unwrapping Method, Accuracy Verification

\begin{abstract}
:
In the 13th Five-Year Plan for Geoinformatics Business, it is proposed that the new InSAR technology should be applied to surveying and mapping production, which will become the innovation driving force of geoinformatics industry. This paper will study closely around the new outline of surveying and mapping and then achieve the TerraSAR/TanDEM data of Bin County in Shaanxi Province in X band. The studying steps are as follows; Firstly, the baseline is estimated from the orbital data; Secondly, the interferometric pairs of SAR image are accurately registered; Thirdly, the interferogram is generated; Fourth, the interferometric correlation information is estimated and the flat-earth phase is removed. In order to solve the phase noise and the discontinuity phase existing in the interferometric image of phase, a GAMMA adaptive filtering method is adopted. Aiming at the "hole" problem of missing data in low coherent area, the interpolation method of low coherent area mask is used to assist the phase unwrapping. Then, the accuracy of the interferometric baseline is estimated from the ground control points. Finally, 1: 50000 DEM is generated, and the existing DEM data is used to verify the accuracy through statistical analysis. The research results show that the improved InSAR data processing method in this paper can obtain the high-precision DEM of the study area, exactly the same with the topography of reference DEM. The $\mathrm{R}^{2}$ can reach to 0.9648 , showing a strong positive correlation.
\end{abstract}

\section{INTRODUCTION}

The Digital Elevation Model (DEM) describes the elevation information of the ground and is widely used in various fields such as engineering earthwork calculation, terrain threedimensional model, auxiliary remote sensing image classification and geological reconnaissance reference. Common DEM mapping methods include traditional field surveys (Tong, 1990), optical remote sensing (Ma, 2009), Light Detection and Ranging (LiDAR) (Liu et al, 2005) and Synthetic Aperture Radar Interferometry (InSAR) (Perna, 2015). The precision of DEM obtained from traditional fieldwork measurement is higher, but the workload, spatial density and time sampling rate of the observation point are difficult to guarantee; Optical remote sensing technology can be carried out on the ground observation of large area with high precision, but the image spatial resolution is limited. And it is very affected by climatic conditions, making it difficult to achieve effective precision (Li and Feng, 2009); LiDAR system has the advantage of obtaining high-precision DEM in difficult surveying areas, but it has some disadvantages such as single data source acquisition and limited single observation area (Liu, 2008). Compared with other topographic surveying methods, the spaceborne InSAR technology has its own unique approach. The imaging process is not affected by complicated weather conditions such as cloud and rain, and has the characteristics of continuously observing the ground information throughout the day (Chen, 2016). With the continuous development of new surveying and mapping, the resolution of spaceborne InSAR system for acquiring image is continuously increasing, the working modes are diversified and the revisiting cycle is shortened. The use of InSAR technology to obtain large-area and high-precision DEMs has shown great potential (Wan et al, 2015; Zhao, 2016; Zhang et al, 2017).

The emergence of InSAR technology can be traced back to the 1960 s, the foreign scholars surveyed in greatly undulate terrain by phase information. Li and Goldstein (1990) used the SEASAT data to analyse the influence of baseline and noise on the accuracy of DEM and proposed the concept of the best baseline. Pascazio (2002) developed the DEM reconstructing method of steep slopes and discontinuous terrain. Fornaro (2005) made centimeter-level DEM generation using multi-baseline joint InSAR data. In 2007, DLR successfully launched the first $\mathrm{X}$-band radar remote sensing satellite TerraSAR-X which is providing a high-resolution, short-revisited data source for the in-depth research and application of InSAR technology. In 2010, the DLR launched a TanDEM-X satellite again in X-band and formed the formation flight mode with the former TerraSAR-X satellite. The data acquired by the two satellites are synchronized in time and space, making up the defect of conventional InSAR data with time-space baseline decorrelation, and providing an effective guarantee for the fast and large-scale extraction of global high-precision DEM data. Since then, many scholars began the researching work of DEM and the evaluation of accuracy based on the satellites flown by

\footnotetext{
${ }^{*}$ Corresponding author
} 
TanDEM-X and TerraSAR- $X$ and achieved good results (Chen et al, 2015; Du, 2015; Sun, 2016).

In this study, the GAMMA software is used to estimate the baseline from the acquired TerraSAR binary orbital data, and then performing the fine (sub-pixel) registration, generating the interferogram, estimating the coherent coefficients of the interferogram, removing the flat-earth phase, doing the adaptive filtering of the interferogram, phase unwrapping, finally estimating the accuracy of the interferometric baseline from ground control points. In order to deal with the problem of phase noise and phase discontinuity existing in the phase image, a GAMMA adaptive filtering method is adopted. In order to solve the "hole" problem of missing data in low coherent area, phase unwrapping is assisted in the interpolation method of low coherent mask, high-precision DEM is final generated.

\section{TEST SITE AND DATA}

\subsection{The general situation of test site}

In this paper, Bin County in Shaanxi Province is chosen as the study area. The geographical coordinate ranges from $34^{\circ} 54^{\prime} 18$ "-35 ${ }^{\circ} 28^{\prime} 16^{\prime \prime}$ in north latitude and $107^{\circ} 52^{\prime} 59^{\prime \prime}-108^{\circ} 21^{\prime} 18^{\prime \prime}$ in east longitude, the detailed location is shown in Figure 1. Bin County is located in Longdong hills and gully areas of Loess Plateau, the topography is high in southwest and low in northeast. Jing River run through the middle area from northwest to southeast, and then the geomorphology in county area is divided into northeast and southwest plateau with roads in the middle. The experimental data covers about $90 \%$ of the administrative area including Bin County. The main landforms are hills, mountains, and a small part of the rivers and plains.

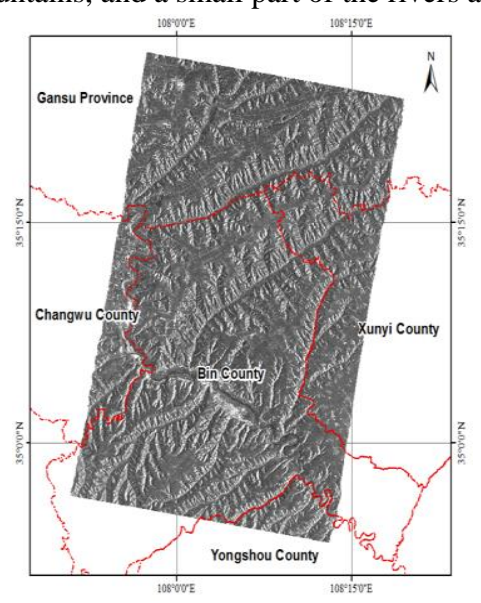

Figure 1. Range of the test site

\subsection{Data Source}

\section{(1) TSX/TDX Data}

The TerraSAR-X images with 3meter resolution in the experimental area would be acquired. A single-track dualantenna system was formed by the formation of TerraSAR-X (TSX) and TanDEM-X (TDX) formations. TerraSAR-X satellite could get the images of high resolution in any area, any time and any weather which are desired by users. Since most of the common interferometric SAR systems are monitoring repetitive orbit interferometry, although better DEMs have been obtained, they still suffer from time and space-related effects as well as atmospheric delays and orbital errors. Comparing with the traditional InSAR system, the most important feature of the TSX/TDX binary satellite distributed SAR system is the ability to obtain "0" time baseline and high-precision orbit interferometric pairs, which can well overcome the decorrelation, atmospheric delays and orbital errors. In addition, the baseline of the TSX / TDX is directly measured by on-board GPS and can be flexibly adjusted and measured in real time, with accuracy that is more accurate than the baseline of a conventional SAR satellite (centimeter-level) to millimeter.

\section{(2) DEM data}

In order to verify the accuracy of the experimental results, the study also obtained nine archival 1: 50,000 DEM data corresponding to the range of TerraSAR data, which is 10 meter in grid resolution. They are I49E007001 、I49E007002 、 I49E006001 、I49E006002 、I49E005001 、I49E005002 、 I48E007024、I48E006024、I48E005024 in details.

\section{RESEARCH METHODS}

\subsection{Fundamentals of DEM extraction by TSX/TDX}

The SAR data used in this paper is obtained under the bistatic mode, as shown in Figure2 (a). In this mode, the signal is transmitted by one of the satellites and received by the two satellites. The two echo signal phase difference analysis to obtain the topographic information. At the same time, the time base of SAR image pairs in bistatic mode is almost zero, which can largely avoid the influences of atmospheric and temporal decorrelation and orbit on the interferometric phase, and facilitate the interpretation of interferometric phases to obtain accurate topographic information.

Figure2 (b) shows the interferometric principles of the TSX/TDX radar satellite system. Where TSX is the TerraSAR$\mathrm{X}$ satellite, TDX is the TanDEM-X satellite, $\mathrm{B}$ is the baseline length between the two, $\mathrm{Bx}$ is the component of the baseline $\mathrm{B}$ in the horizontal direction, $\mathrm{Bz}$ is the component of the baseline $\mathrm{B}$ in the vertical direction, $\mathrm{P}$ is any point on the surface, $\theta$ is the incidence angle of the main image, $\alpha$ is the angle between the vertical baseline and the horizontal direction, $r_{1}$ and $r_{2}$ are the distances from the ground point $\mathrm{P}$ to the two SAR sensors respectively, $h$ is the elevation of point $\mathrm{P}$, and $\mathrm{H}$ is the distance between satellite and ground.

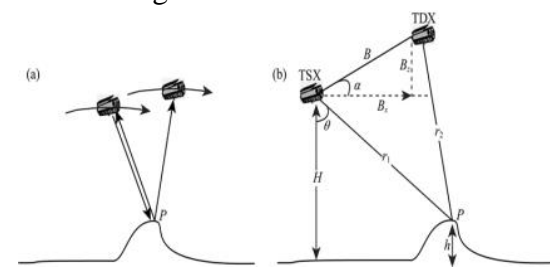

Figure 2. Radar system interference principle diagram 


\subsection{Multilook processing}

Single-Look Complex (SLC) data is the original highest resolution of SAR data. The coherent addition of single-cell backscattering information will result in a lot of noise in the intensity information. Multilook processing during image focusing can be applied to improve radiometric accuracy at the cost of image resolution. And then get the intensity data. In this paper, the multilook program is used to process the SLC data and transforms it into multi-look intensity data. The multi-look number of azimuth and distance is determined according to the original imaging ranks to be $3 * 3$. The multilook SAR intensity image is shown in Figure 3.

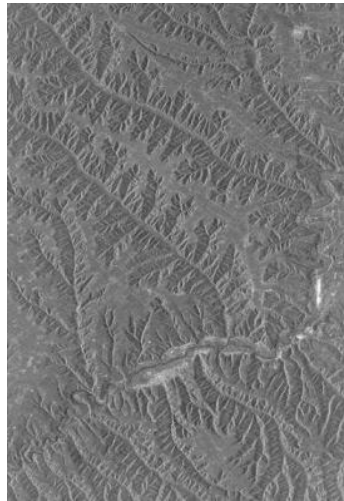

(a)tdx.mli

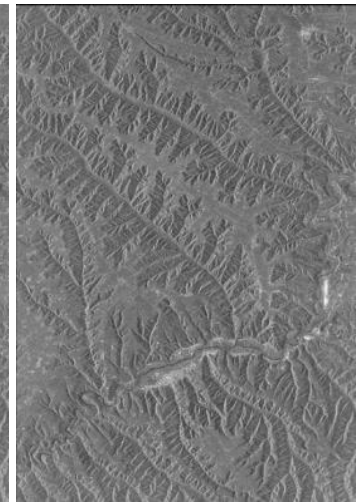

(b)tsx.mli
Figure 3. TDX/TSX Multi-intensity Image

\subsection{Fine registration}

The sub-pixel registration of both focused SAR images is a strict requirement for interferometric processing. Just and Bamler have shown that coregistration to an accuracy of $1 / 8$ th of a pixel yields an almost negligible decrease in coherence, as long as the data are acquired with relatively small squint angles (Just and Bamler,1994). In this paper, create_offset is used to create the offset parameter file, and then init_offset is used to estimate the offset of two SAR images.

In order to get a more accurate estimate, the procedure of init_offset is used in multi-looking image firstly and then in a single image secondly. On the basis of the initial offset, the accurate estimation of the offset polynomial can be further performed, which can be based on the intensity image or the SLC image. Finally, the offset in range direction and azimuth direction are 0.032 and 0.005 pixels respectively, which meet the requirement of interference processing.

\subsection{Interferogram formation}

A complex interferogram is constructed by a pointwise complex multiplication of corresponding pixels in both datasets, the complex interferogram is usually retained for future computations. In this paper, the procedure of interf_SLC is used to do the processing, and the local area is shown in Figure4.

As you can see in Figure4, the stripes are more densely affected by the flat-earth phase. In addition, the phase noise is more serious where there is a terrain gradient, so the subsequent processing of interferogram need to be performed on removing the flat-earth phase and filtering.

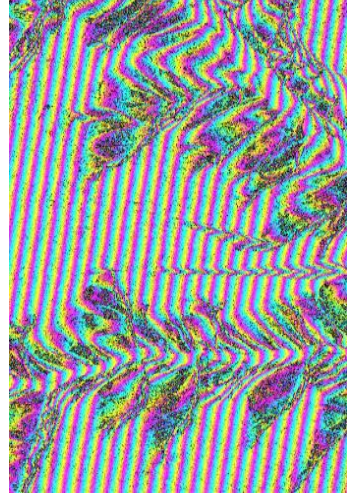

(a)

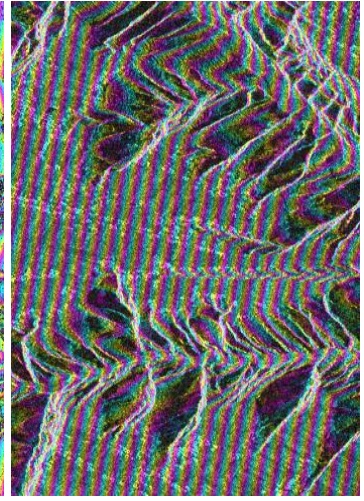

(b) (a) Interferogram; (b) Interferogram overlay intensity image Figure 4. Interferogram results

\subsection{Flat-earth phase removal}

The settled height of the flat surface will appear interferometric fringes which is changing periodically in the range direction and azimuth direction periodically, and then causing the most complicated result of the interferogram, which is not conducive to follow-up processing. So the interferometric signals multiplied by complex phase function is used to solve this problem. First, the interferometric baseline should be estimated; then, using the baseline file to remove the flat-earth phase, the interferogram after removing the flat-earth phase is shown in Figure5.

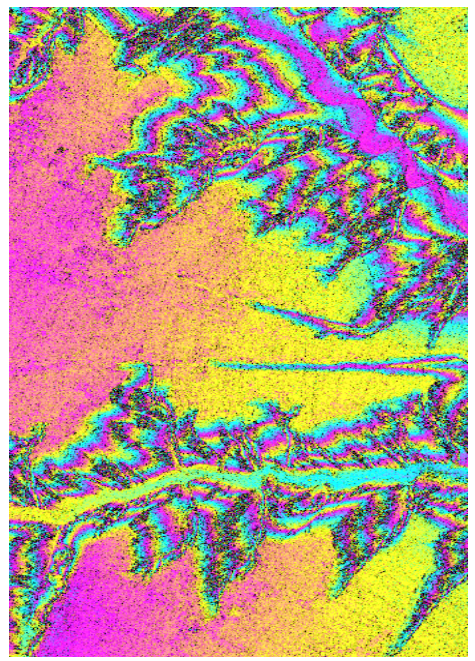

Figure 5. Interferogram after removing flat-earth phase

\subsection{Coherence coefficient calculation}

Based on the filtered interferogram, the interferometric coherence coefficient can be calculated, and the coherence coefficient image can be used in the subsequent filtering or unwrapping process. The displaying effect of the coherence coefficient is shown in Figure6. It can be seen that the coherence of flat ground is better, while the coherence of the terrain slope area is relatively poor. 


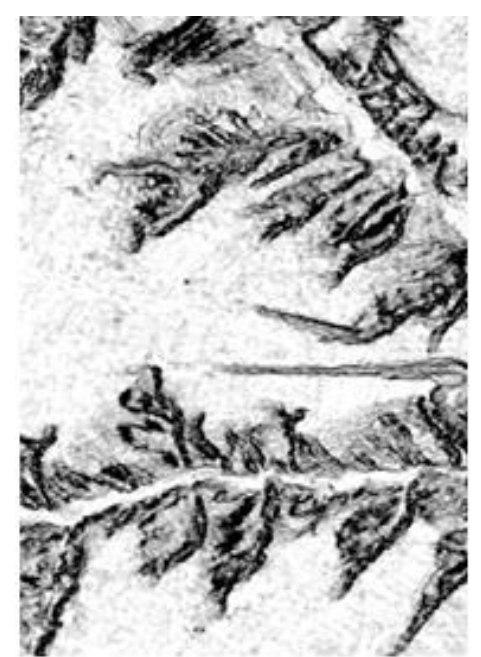

Figure 6. Coherence coefficient image

\subsection{Adaptive Filtering}

Due to the presence of a certain phase noise in the interferogram, this results a certain error in phase unwrapping. Therefore, before the phase unwrapping, we need to smooth the interferogram by filtering firstly. Because of the window which is too small will remove the noise with a bad result, and the large window may introduce a greater unwrapping error. The best adapting window is chosen in this paper. The interferogram after filtering is shown in Figure7.

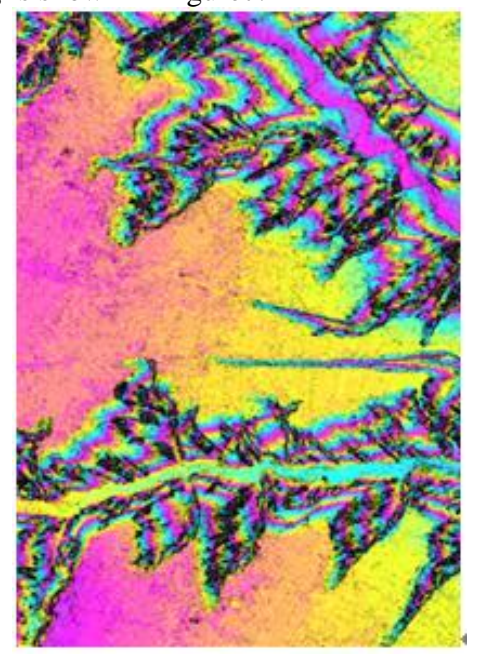

Figure 7. Interferogram after adaptive filtering

\subsection{Phase unwrapping}

Two methods are used in Gamma software which are branchcut and minimum-cost flow. In this study, the method of branch-cut is used to perform phase unwrapping. Compared with the latter method, the unwrapping process needs less computation and space. Branch-cut method consists of four steps: corr_flag, residue, tree_cc, and phase grasses. The images after unwrapped phase may be exist some holes in the lowcoherence area, in order to solve this problem, it can be handled by interpolation (interp_ad). The interferogram after phase unwrapping is shown in Figure8.

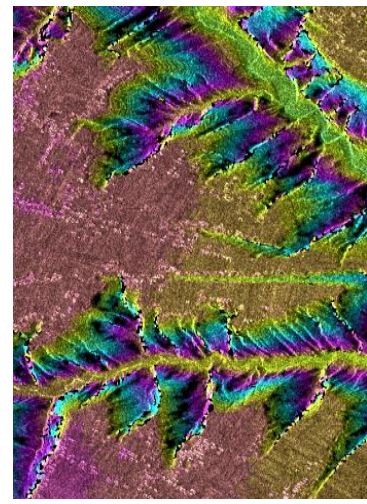

(a) Before interpolation

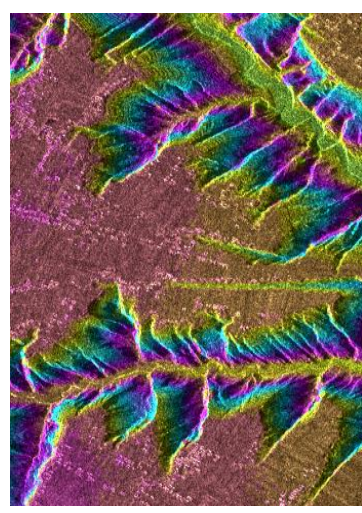

(b) After interpolation Figure 8. Interferogram after phase unwrapping

\subsection{Precision Estimation of the Baseline}

The accuracy of the initial estimate of the interferometric baseline of the flat-earth phase is not sufficient for elevation calculations. Therefore, the control of ground control points requires a more precise machine selection estimation, which mainly includes three steps: a. Selecting the ground control points; b. Extracting the elevation and phase of the ground control points; c. Least squares estimation of the interferometric baseline. In order to facilitate the selection of ground control points, this study completed the geocoding of SAR images at this step, and established the relationship between SAR images and geospatial space.

And then the control points should be evenly distributed throughout the image, it is recommended to select at least 20 control points. Based on the control point information, a more accurate estimate of the baseline is carried out. The elevation image calculated by phase is shown in Figure 9.
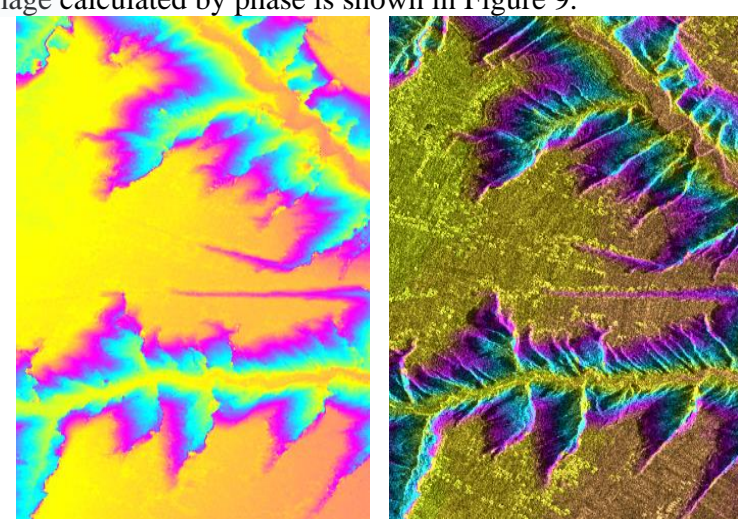

(a)Elevation image (b)Elevation overlay intensity image Figure 9. Elevation image of local area

\section{RESULTS AND ACCURACY VERIFICATION}

\subsection{Results}

Using the look-up table file in the geocoding process, the elevation file obtained by InSAR could be sampled into the geographic coordinate space, and the geocoded elevation image is shown in Figure10. 


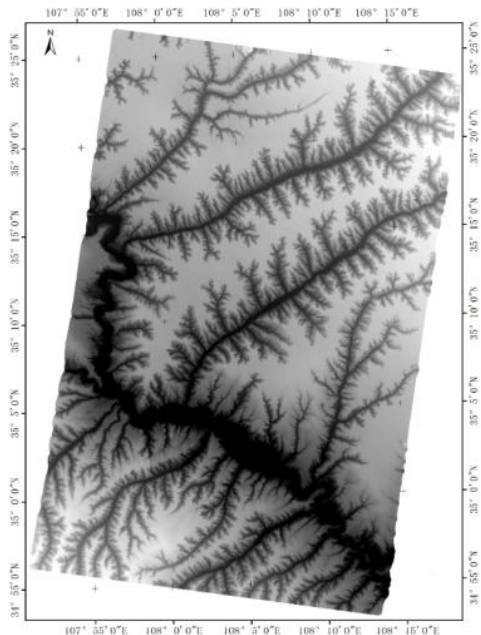

Figure 10. Geocoded DEM

\subsection{Accuracy Verification}

For more scientific and accurate evaluation of the elevation accuracy of DEM, 1:50000 DEM data acquired from survey department was used as the reference DEM to test the results. Since a small part of area in north of Bin county covered by SAR image is outside of the Shaanxi province, the 1: 50000 DEM acquired in this study could not completely cover all the SAR image ranges. Therefore, only the inner provincial part is intercepted for the accuracy verification. The specific area is as shown in Figure11. In the experimental area, different topographic conditions and number of different coherency checkpoints are selected uniformly. The distribution of checkpoints is shown in Figure11(c).

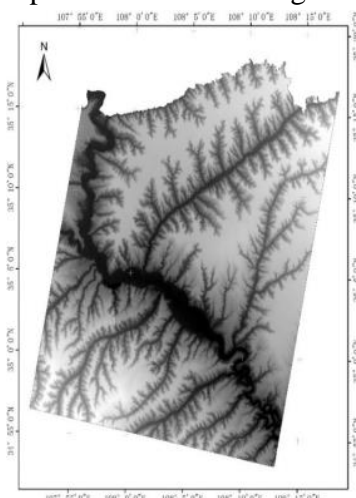

(a) InSAR DEM

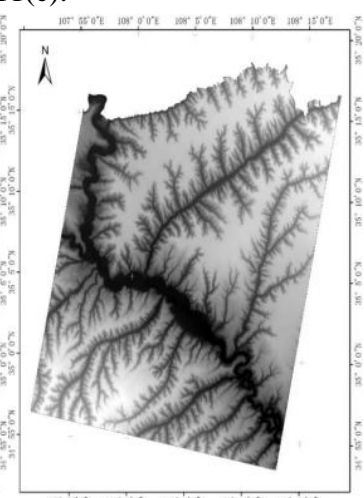

(b) 1:50000 DEM

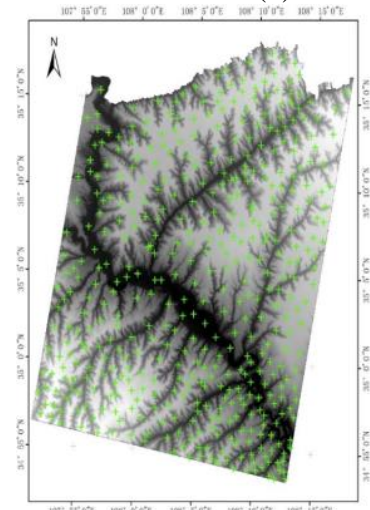

(c) Checkpoint distribution

Figure 11. The registered InSAR DEM and 1: 50,000 DEM
Then the elevation values of each checkpoint of the same name are extracted respectively from the result DEM and the reference DEM. The precision evaluation results were obtained by statistical analysis. The reason of the difference in accuracy of the interference results under different topographic conditions has been analysing in detail.

In the study, the correlation and mean square error of height are as two indicators to test the accuracy. First, correlation analysis was used to verify the correlation between InSAR DEM and 1: 50000DEM. The correlation analysis results are shown in Figure 12. As can be seen from the figure, the research results have a very good linear correlation with the reference DEM, R2 can reach 0.9648 , showing a strong positive correlation, which shows that the InSAR interference results are highly consistent with the reference DEM topography. Secondly, the deviation of InSAR DEM and elevation of 1: 50000 DEM has been analysing by means of the same precision detection method to calculate the error in the elevation, and the error in the obtained elevation was 6.0 meters. The result shows that the method of InSAR interference in this study can be basically meet the accuracy requirement of 1: 50000 DEM drawing.

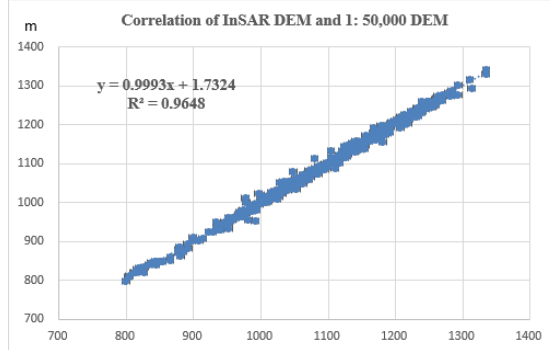

Figure 12. Correlation of InSAR DEM and 1: 50,000 DEM

Combined with the actual terrain in the experimental area, the analysis of the overall error of the checkpoint shows that the larger deviations are mainly distributed in the mountainous areas. The reason is that the mountainous terrain has a large topography with a small amount of vegetation coverage and poor coherence. As a result, the elevation error in the area is relatively large. On the other hand, the ups and downs of elevation in the mountainous area are obvious, and the slight difference of the plane precision causes certain elevation error. Flat areas, farmland, roads and other distribution more flattened, more coherent, so the elevation accuracy is relatively high.

\section{CONCLUSION AND DISCUSSION}

This paper is studying based on the TSX/TDX data, GAMMA software is used to read the original data to generate SLC primary and secondary image interferometric pairs, and the two pairs of SAR data are converted to intensity images by multilook processing. On this basis, accurate coregistration of two images, generation of interferogram, flat-earth phase removal, estimation of coherence coefficient, adaptive filtering of interferogram, phase unwrapping, accurate estimation of interferometric baseline from ground control point, and finally phase to elevation conversion have been done. In the end, the results in SAR Doppler coordinate system is transformed to the WGS84 coordinate system by geocoding, and the grid interval of DEM is resampling to 10 meters in the same time.

In the fine registration process, the offsets in range direction and azimuth direction are 0.032 and 0.005 pixels respectively, which are achieving the sub-pixel level registration accuracy 
required by the interferometric process. In the process of filtering and phase unwrapping, this paper selects the best filtering and unwrapping algorithm according to the actual topography condition of Bin county experimental area, effectively avoids the error in subsequent processing and greatly improves the accuracy of elevation calculation and efficiency. The research results have a very good linear correlation with the reference DEM, R2 can reach to 0.9648 , and the mean square error of height is 6.0 meters, which can meet the accuracy requirement of 1: 50000 DEM mapping.

\section{ACKNOWLEDGEMENTS}

This research work would not have been done well without the help of Dr.Zhao in Chinese Academy of Forest, the author will be grateful to the assistance of data processing and software supporting.

\section{REFERENCES}

Chen, P.Q., Long, S.C., Jiang, Z.L., Yuan, Y., 2015. Study of InSAR-derived DEMs generated with TanDEM $-X$ and ERS Tandem Data. Journal of Hunan University of Science \& Technology (Natural Science Edition), 30(4), pp. 21-26.

Chen, W.N., 2016. Study on DEM generating method by Spaceborne SAR. Xi'an University of Science and Technology.

Du, Y.N., Feng, G.C., Li, Z.W., Zhu, J.J., Peng, X., 2015. Generation of high precision DEM from TerraSARX/TanDEM-X. Chinese Journal of Geophysics, 58(9), pp. 3089-3102.

Ma, S.Y, 2009. Research on the Extraction of DEM Based on High Resolution Remotely-sensed Data. East China Normal University.

Fornaro, G., Lombardini, F., Serafino, F, 2005. Threedimensional multi-pass SAR focusing: Experiments with longterm spaceborne data. IEEE Transactions on Geoscience and Remote Sensing, 43(4), pp. 702-714.

Just, D., Bamler, R., 1994. Phase statistics of interferograms with applications to synthetic aperture radar. Applied Optics, 33(20), pp. 4361-4368.

Li, F.K., Goldstein, R.M., 1990. Studies of multi-baseline spaceborne interferometric synthetic aperture radars. Geoscience and Remote Sensing, IEEE Transactions on, 28(1), pp. 88-97.

Liu, X., Peterson, J., Zhang, Z., 2005. High-resolution DEM generated from LiDAR data for water resource management. In: Proceedings of the International Congress on Modelling and Simulation (MODSIM05). Modelling and Simulation Society of Australia and New Zealand Inc, pp.1402-1408.

Liu, X., 2008. Airborne LiDAR for DEM generation: some critical issues. Progress in Physical Geography,32(1), pp. 31-49.

Li, Z., Feng, Y., 2009. Geometric Model of Optical Spaceborne Sensors and DEM Generation. Geomatics World, (02), pp. 5362.
Pascazio, V., Schirinzi, G., 2002. Multifrequency InSAR height reconstruction through maximum likelihood estimation of local planes parameters. IEEE Transactions on Image Processing, 11(12), pp. 1478-1489.

Perna, S., Esposito, C., Berardino, P., Pauciullo, A., Wimmer, C., Lanari, R., 2015. Phase offset calculation for airborne InSAR DEM generation without corner reflectors. IEEE Transactions on Geoscience and Remote Sensing, 53(5), pp.2713-2726.

Wan, L., Zhou, C.X., E, D.C., Deng, F.H., 2015. DEM generation and precision analysis of Antarctic ice sheet based on InSAR and ICESat data. Journal of Glaciology and Geocryology, 37(5), pp. $1160-1167$.

Sun, Y.F., Jiang, L.M., Liu, L., Sun, Y.L., Wang, H.S., 2016. Generation and evaluation digital terrain model with TanDEM$\mathrm{X}$ bistatic SAR interferometry. Geomatics and Information Science of Wuhan University, 41(1), pp. 100-105.

Tong, S.Q., 1900. Field sampling density and precision of digital elevation mode. Journal of PLA surveying and mapping institute, (01), pp.63-68.

Zhang, L.Q., Liu, H.Y., Guo, Y.K., Liao, B., 2017.The methodology on building high- fidelity DEM in road domain based on multi-source Data. Bulletin of Surveying and Mapping, (7), pp. 113-116,142.

Zhao, Z., 2016. Methods on high-accuracy DEM extraction from interferometric SAR in sophisticated terrain areas. Acta Geodaetica et Cartographica Sinica, 45(11), pp. 1385. 